\title{
Echocardiogram in Pulsus Paradoxus
}

\section{Respiration Dependent Cyclic Changes in Mitral and Aortic Valve Motion: A Case Report}

\author{
Tsuguya Sakamoto, M.D., F.A.C.C., Chuwa Tei, M.D., \\ Terumi Hayashi, M.D., Hirofumi IchiYasu, M.D., \\ Keiko Amano, M.D., Yoshiyuki Hada, M.D., \\ and Tsuyoshi Serizawa, M.D.
}

\section{SUMMARY}

The mechanism of production of pulsus paradoxus was echocardiom graphically studied in a 74-year-old male with subacute effusive-constrictive pericarditis which developed to constrictive pericarditis under the observation. Echocardiography disclosed the following phenomena during inspiration: 1) mitral valve did not open until the atrial systole, probably because of the lack of antegrade mitral flow during rapid filling phase (the $\mathbf{E}$ wave was not observed), 2) concomitantly, aortic valve opening decreased markedly in its grade, and 3) left ventricular ejection time (LVET) decreased and pre-ejection period (PEP) increased, resulting in a higher PEP/LVET ratio (up to 1.32). The opposite was true during expiration (PEP/LVET ratio was 0.40$)$.

This is probably the first case, in which the mechanism of pulsus paradoxus was investigated by aortic and mitral valve echograms.

\section{Additional Indexing Words:}

Echocardiography Systolic time intervals (STI) Effusive-constrictive pericarditis Constrictive pericarditis

7 HE mechanism of production of pulsus paradoxus has been discussed by 1 many previous authors with equivocal explanation. ${ }^{1)-7)}$ Recently, the use of echocardiography has enabled us to study the movement of cardiac structures including pericardial abnormalities, ${ }^{9 /-12}$ and $D^{\prime} \mathrm{Cruz}$ et al ${ }^{8)}$ discussed the problem of pulsus paradoxus in cardiac tamponade. However, the present authors experienced a case, in which the pulsus paradoxus occurred with abnormal valve movements under the observation of subacute effusive-constrictive pericarditis. ${ }^{14)}$ The purpose of this report is to describe an interesting valve mechanics found in this case, and to present the possible and heretofore undescribed mechanism of pulsus paradoxus.

From the Second Department of Internal Medicine, Faculty of Medicine, University of Tokyo, Hongo 7-3-1, Bunkyo-ku, Tokyo 113, Japan.

Received for publication April 15, 1977. 


\section{Gase Report}

A 74-year-old male was admitted to the Tokyo University Hospital in September, 1975, because of progressive palpitation, breathlessness, precordial discomfort, slight fever, and night sweat. He had complained of cough for 1 year. He had been pointed out cardiomegaly at a local hospital 3 months ago. At the time of admission, the physical examination revealed that the radial arterial pulse was not palpable during inspiration. The cardiac rhythm was regular with a rate of 80 per minute. The blood pressure was $118 / 100 \mathrm{mmHg}$ with a decreased pulse pressure. The jugular veins were prominently engorged. The pitting edema was noted at the pretibial region. On auscultation, dry râle was heard and expiratory phase was prolonged. Heart sound was distant and gallop sound was not heard. There was no murmur. The hepatic edge was palpable $5 \mathrm{~cm}$ below the right costal margin. There was no ascites. Venous pressure increased to $260 \mathrm{~mm}$ $\mathrm{H}_{2} \mathrm{O}$.

Laboratory studies gave the following results. The erythrocyte sedimentation rate was $8 \mathrm{~mm} / \mathrm{h}$, the concentration of hemoglobin $13.7 \mathrm{Gm} / 100 \mathrm{ml}$ of blood, the hematocrit $42 \%$. There was mild elevation in levels of serum GOT, total bilirubin, urea nitrogen, and creatinine. The other biochemical data were within normal limits. Au-antigen and $\alpha$-fetoprotein were negative. Mantoux test was strongly positive. The electrocardiogram (Fig. 1) showed low voltage and non-specific ST-T changes, suggesting pericardial effusion. The chest roentgenogram (Fig. 2) revealed enlarged cardiac silhouette, marked bilateral pleural effusion and right middle lower lobe collapse.

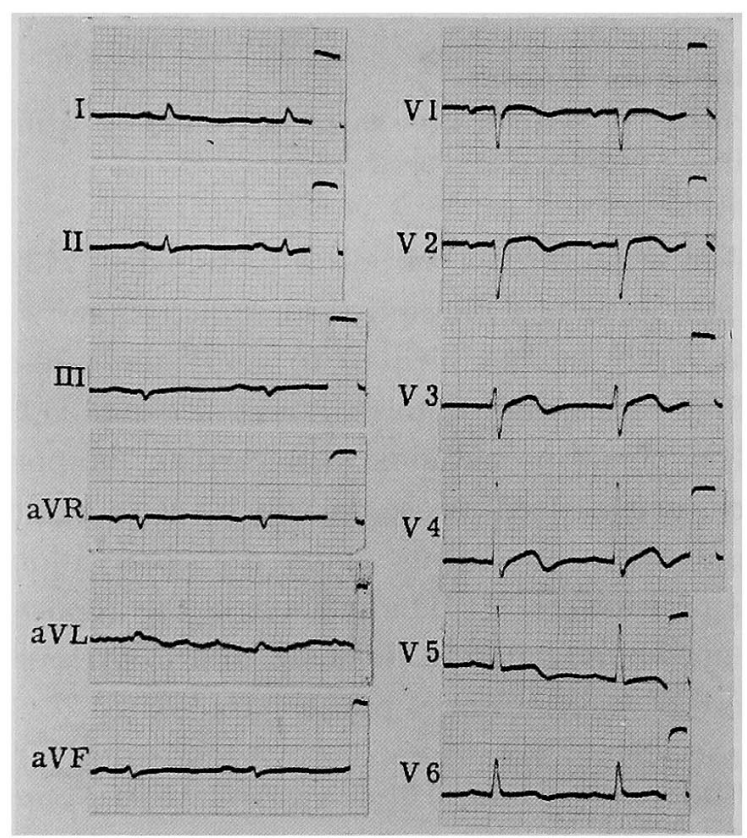

Fig. 1. Electrocardiogram on admission (Sept., 1975). 


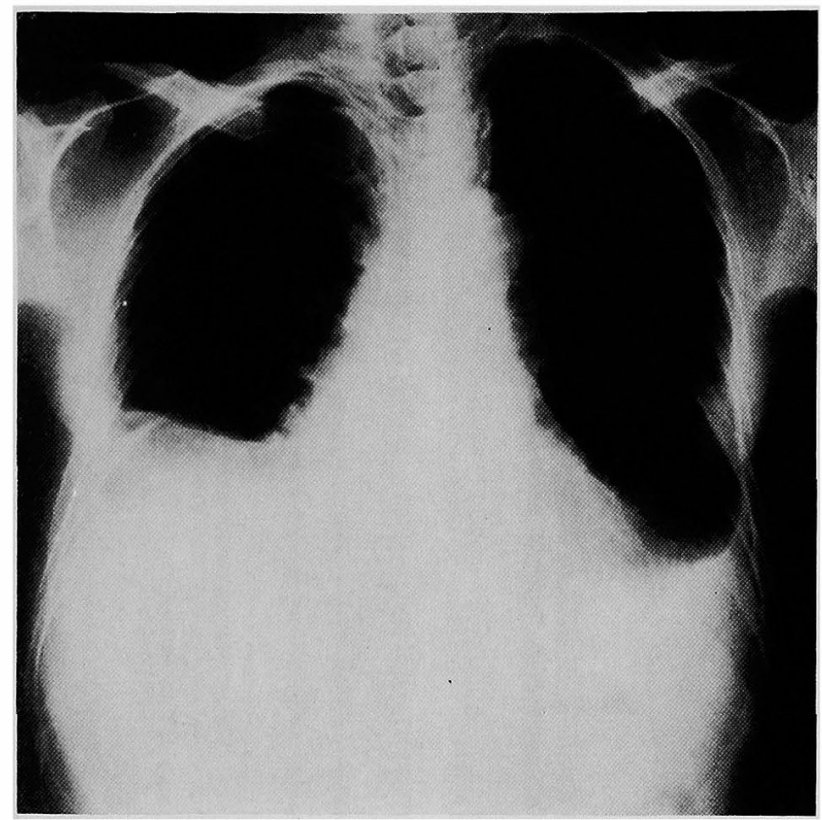

Fig. 2. Chest roentgenogram on admission (Sept., 1975).

Fig. 3 shows the echocardiogram examined on admission. The systolic aortic valve opening and separation (Fig. 3 upper) became decreased markedly on inspiration during respiration. The pre-ejection period (PEP: from the beginning of the QRS complex to the aortic valve opening) increased during inspiration. On the other hand, the left ventricular ejection time (LVET: from the aortic valve opening to its closure) decreased during inspiration.

Fig. 4 shows respiratory changes of left-sided systolic time intervals (STI) of consecutive 18 heart beats ( $R-R$ intervals did not change throughout the whole cycles). At the end of inspiration, PEP is markedly prolonged than LVET, resulting in a higher PEP/LVET ratio (up to 1.32). The respiratory phasic changes of the aortic valve separation was in parallel with LVET. The isovolumetric contraction time (ICT: from the mitral valve closure to the aortic valve opening) showed the respiratory alteration, so did the PEP. Furthermore, Q-IIA interval revealed less respiratory change in comparison with PEP, LVET, PEP/LVET and ICT, and slightly decreased during inspiration and increased during expiration.

The mitral valve (Fig. 3 lower) did not open until the atrial systole, probably because of the lack of antegrade mitral flow during inspiration. The $\mathbf{E}$ wave was observed only during expiration. The diastolic descent rate (DDR) of the anterior mitral valve decreased $(48 \mathrm{~mm} / \mathrm{sec})$.

The interventricular septum (Fig. 5) revealed the typical paradoxical movement. The flattening of the mid to late diastolic phase in the left ventricular endocardium and epicardium was observed. Left ventricular dimension decreased at both end-diastole and end-systole $(31 \mathrm{~mm})$. The epicardium was thickened and showed a strong band of echo. There was an echo free space between epicardium and pericardium. Pleural effusion was also revealed behind the pericardium. 


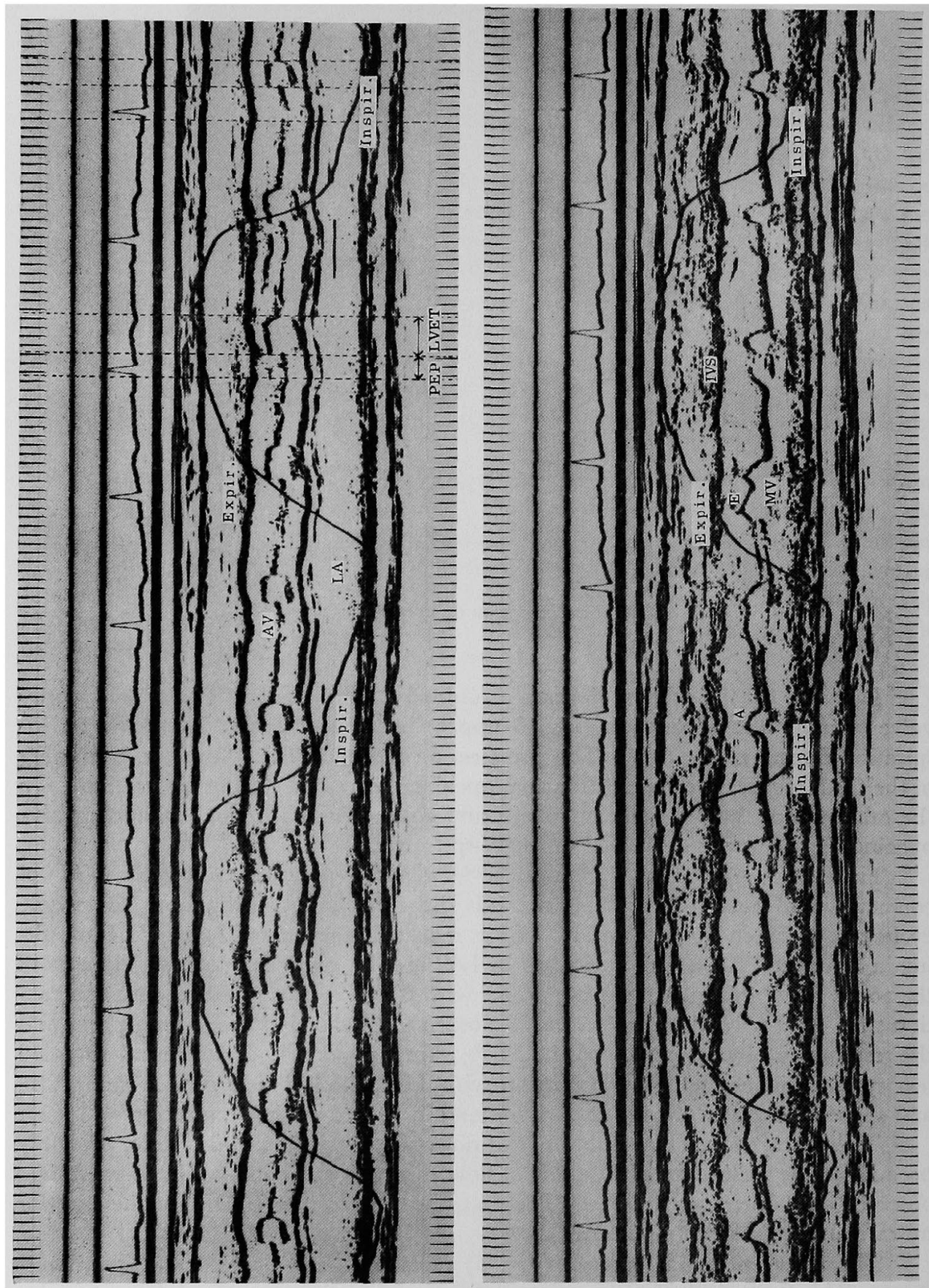

Fig. 3. Respiratory cyclic changes in aortic and mitral valve echogram recorded on admission. Upper: aortic valve opening or separation decreased markedly during inspiration. PEP is longer than LVET during inspiration. Lower: the mitral valve does not open until atrial systole during inspiration. The $\mathbf{E}$ wave of the anterior mitral valve appears only during expiration. PEP: pre-ejection period, LVET: left ventricular ejection time. 


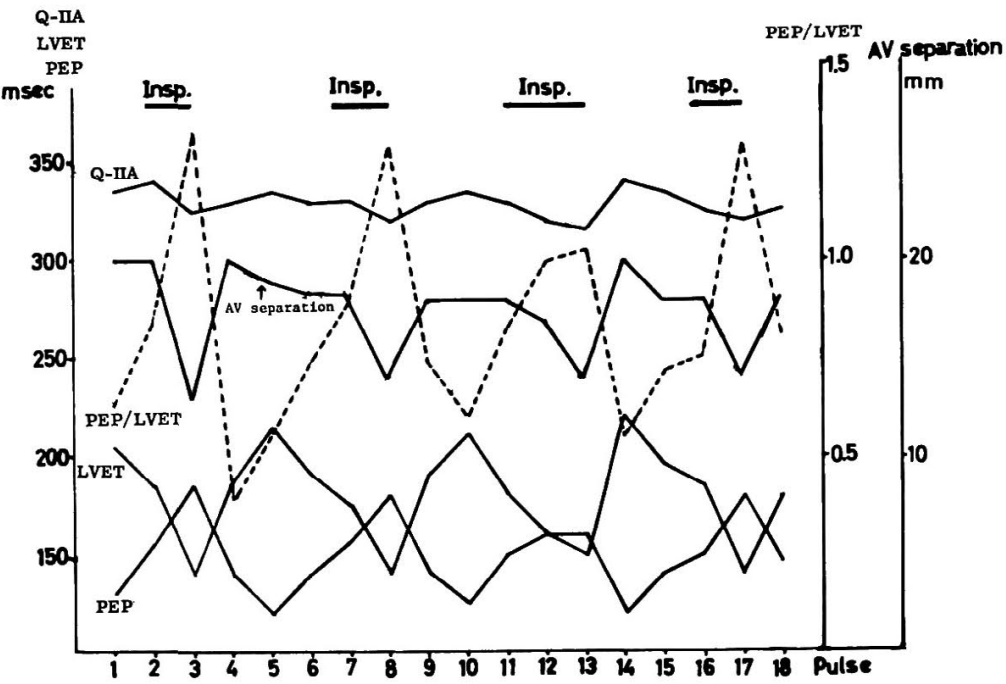

Fig. 4. Respiratory changes in left-sided systolic time intervals (STI) and aortic valve (AV) separation in sequential 18 heart beats. See text.

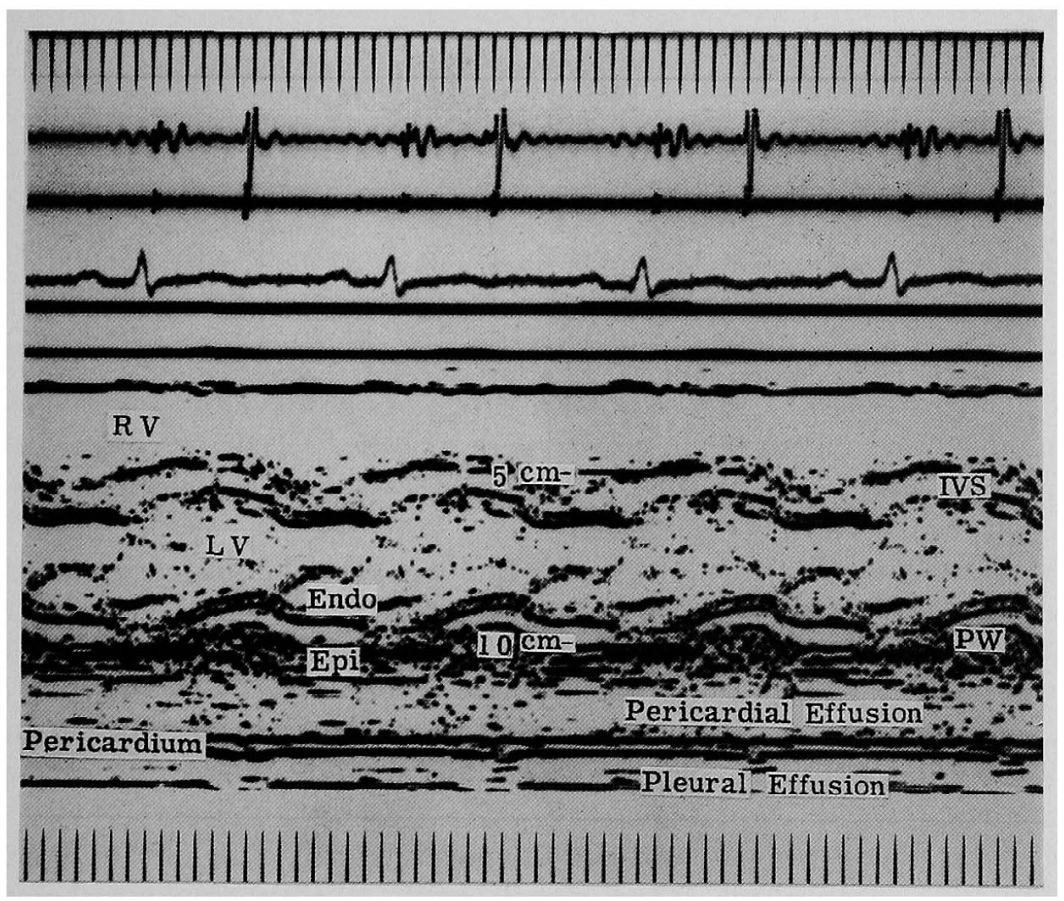

Fig. 5. Echocardiogram of left ventricular short axis direction recorded on admission. The paradoxical movement of the interventricular septum(IVS) is apparent. Both pericardial and pleural effusion are observed. The epicardial echo is thickened and shows a band of echo. RV: right ventricle, LV: left ventricle, PW: posterior wall of left ventricle, Endo: endocardium, Epi: epicardium. 


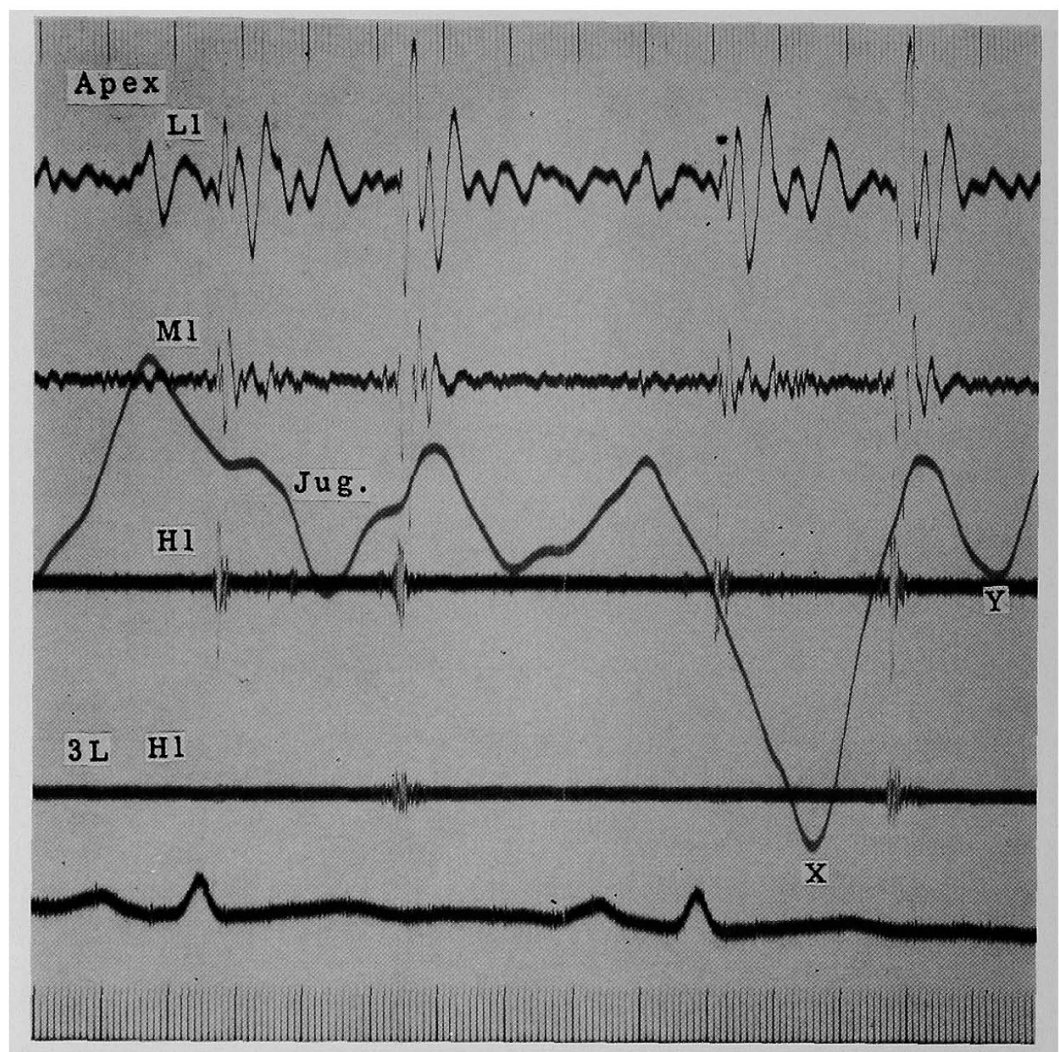

Fig. 6. Phonocardiogram and jugular phlebogram. Neither knock sound nor murmur is recorded. Predominant systolic dip is observed in the jugular phlebogram (Jug.) during expiration (the second beat).

In phonocardiogram (Fig. 6), both first and second heart sounds decreased and faint third and fourth heart sounds were recorded. Neither pericardial knock sound nor murmur was recorded. There was predominant systolic dip in the jugular phlebogram during expiration.

The hemodynamic and angiographic findings gave the following results. The atrial pressure was elevated (a: 15, x: 9, v: 5, y: 11, and mean: $13 \mathrm{mmHg}$ ), and the right ventricular pressure was $31 / 13 \mathrm{mmHg}$. The cardiac output was $1.75 \mathrm{~L} / \mathrm{min}$ and cardiac index was $1.22 \mathrm{~L} / \mathrm{min} / \mathrm{M}^{2}$. Angiography showed small cavities of right atrium, right and left ventricles, irrespective of enlarged cardiac shadow, and suggested pericardial effusion. The washout of the contrast medium was delayed markedly.

Following the investigations, the diagnosis of subacute effusive-constrictive pericarditis was established. One month later, anti-tuberculous therapy was started because of the detection of the bacillus by culture of the sputum. Following the therapy, he gradually improved and marked pulsus paradoxus disappeared. He left the hospital in January, 1976.

Figs. 7 and 8 show echocardiograms examined 10 months later (November, 1976). The echocardiogram did not reveal any phasic respiratory changes of left- 


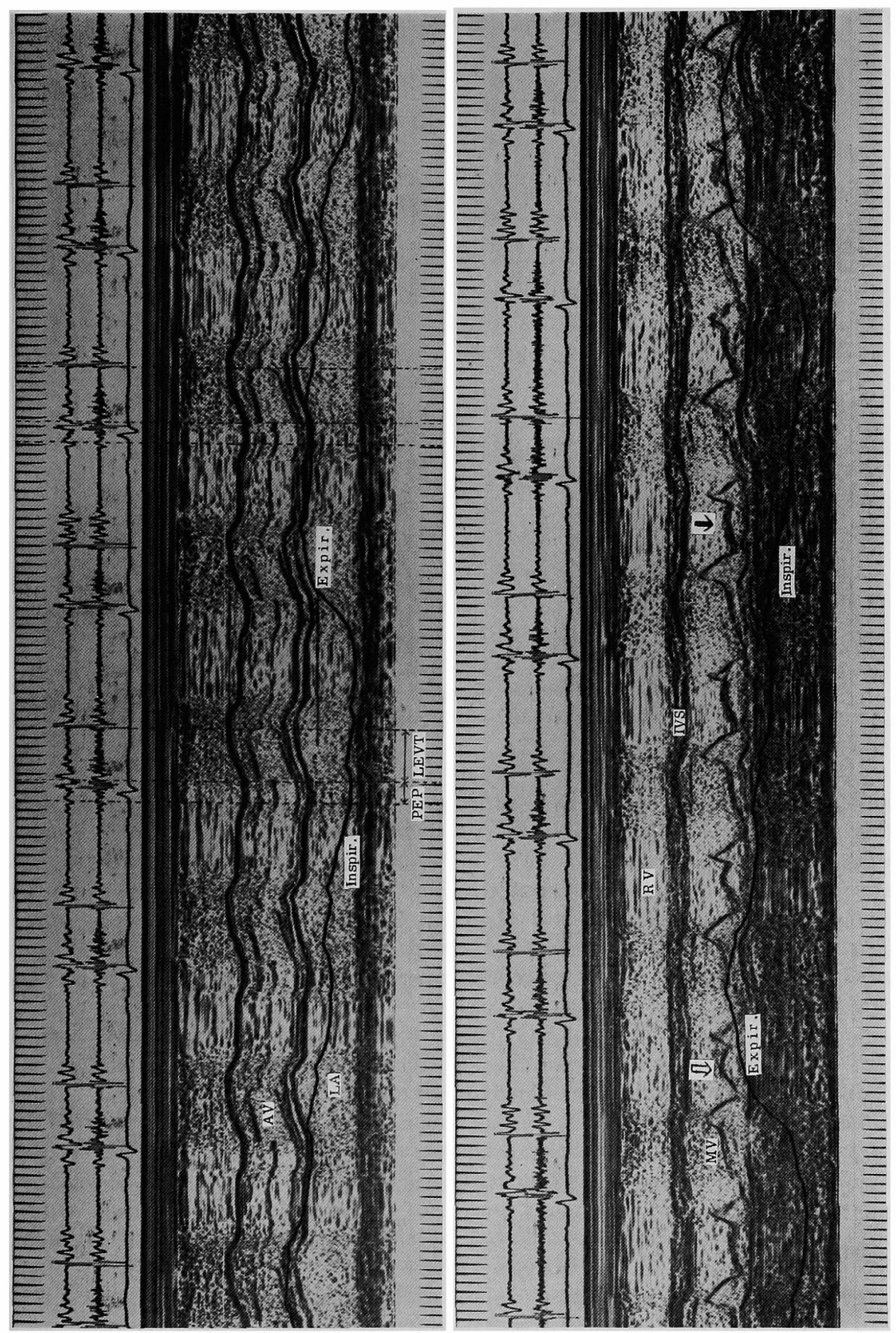

Fig. 7. Aortic and mitral valve echogram after the disappearance of paradoxical pulse. Upper: respiratory change in aortic valve separation and left-sided STI are not shown. Lower: the E wave of mitral valve appears constantly irrespective of the phase of respiration. The mid-diastolic wave of the anterior mitral valve appears with the expiratory exaggeration, which is often observed in cases of constrictive pericarditis. 


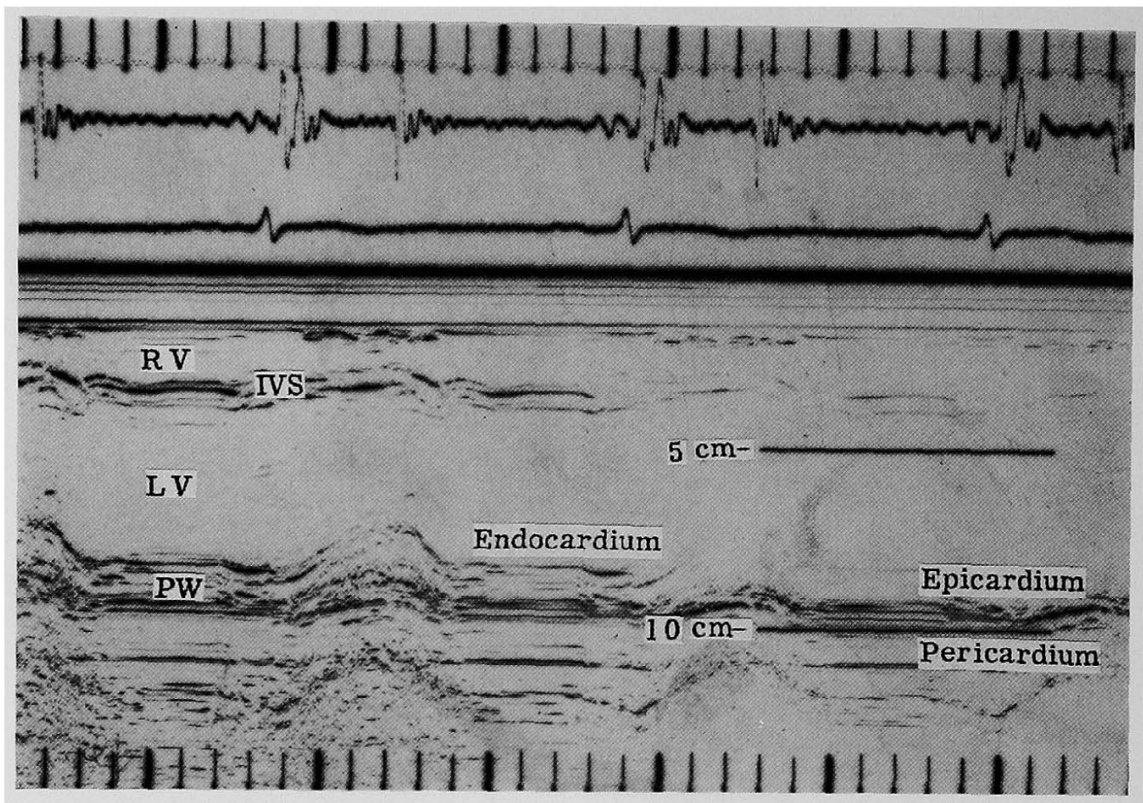

Fig. 8. Echocardiogram of interventricular septum (IVS) and left ventricular posterior wall (PW). The IVS shows paradoxical movement. Both pericardial and pleural effusion disappeared. The motion of $\mathrm{PW}$ between mid and late systole is more flattened. RV: right ventricle, $L V$ : left ventricle.

sided STI and aortic valve separation (Fig. 7 upper). The E wave of mitral valve (Fig. 7 lower) was no longer affected by respiration, and DDR of the anterior mitral valve increased $(150 \mathrm{~mm} / \mathrm{sec})$. The mid-diastolic wave of anterior mitral valve appeared with the expiratory exaggeration. The paradoxical movement of the interventricular septum was still observed, however, the echo free space disappeared (Fig. 8). On the other hand, the flattening of the mid and late diastolic motion of the left ventricular endocardium, epicardium, and pericardium increased (Fig. 8).

Figs. 9, 10, and 11 show EGG, chest X-P and PGG examined in November 1976. In the electrocardiogram (Fig. 9), the low voltage of precordial leads was improved despite the presence of low voltage in the limb leads. The ST-T changes still existed, and $\mathrm{rSr}^{\prime}$ pattern in $\mathrm{V}_{1}$ was shown. The chest roentgenogram (Fig. 10) revealed slightly decreased cardiac silhouette and disappearance of pleural effusion. The jugular phlebogram and phonocardiogram (Fig. 11 upper) showed the evidence of constrictive pericarditis. The $\mathrm{x}$ descent of the venous pulse was shallow and the prominent early y trough was followed by a diastolic plateau. The knock sound also occurred almost coincidentally with y trough. The findings of hepatic pulse (Fig. 11 lower) were almost same to those of the jugular venous pulse.

It was regarded that constrictive pericarditis developed under the observation of subacute effusive-constrictive pericarditis. 


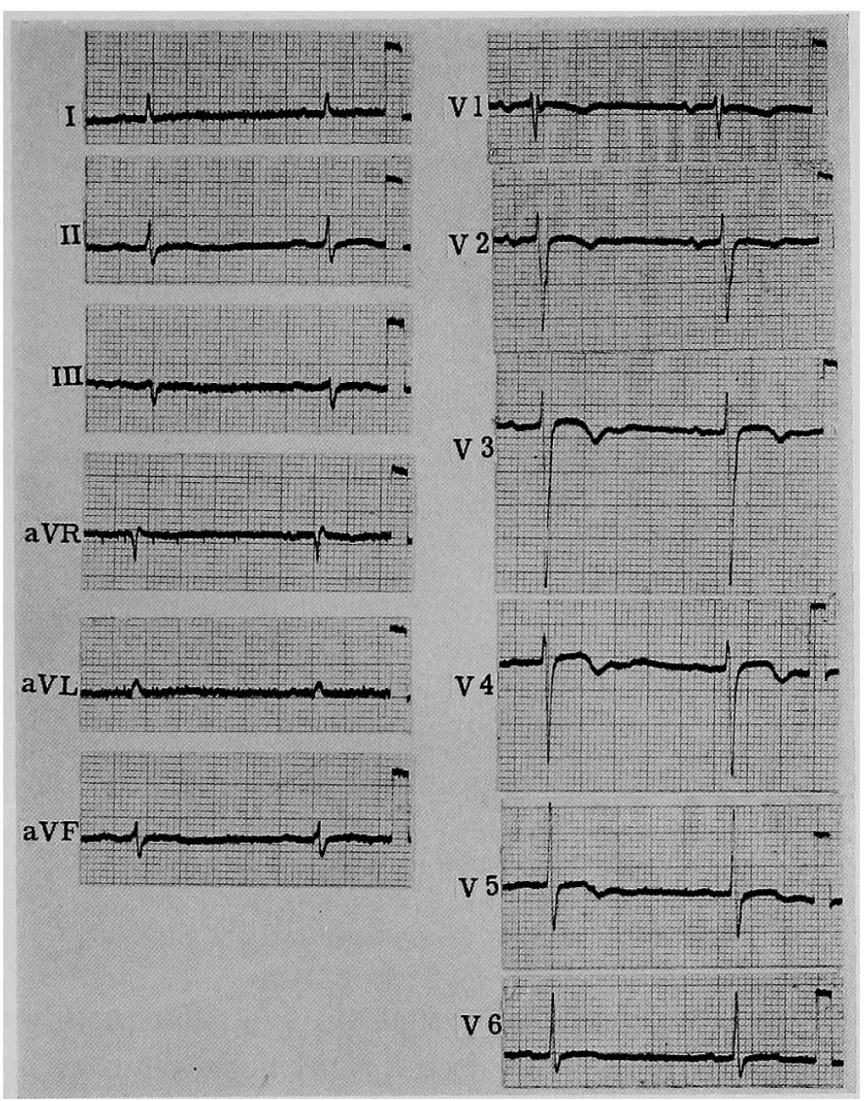

Fig. 9. Electrocardiogram in recovery phase (Nov., 1976).

\section{Discussion}

Several theories have been proposed concerning pulsus paradoxus in pericardial disease. The theory of Katz and Gauchat, ${ }^{1 \prime}$ confirmed by the experiment of acute tamponade by Golinko et al, ${ }^{2)}$ is such that the left ventricular filling decreased during inspiration. This is due to the pressure reversal between left atrium and pulmonary vein, because the intrapericardial pressure does not fall during inspiration in spite of the decrease in the pulmonary venous pressure. The mitral and aortic valve movements seen in this study seem to endorse their theory because of the inspiratory disappearance of the $\mathbf{E}$ wave of the mitral valve. This is compatible with the backward flow to pulmonary vein ${ }^{2)}$ during this phase. Concomitantly, the aortic valve motion also reflects the decreased forward flow and this fact strongly denotes that the echocardiographic sign of the mitral valve is not due to the particular cardiac motion frequently observed in pericardial effusion. The change in STI also indicates the striking respiratory variation of stroke volume compat- 


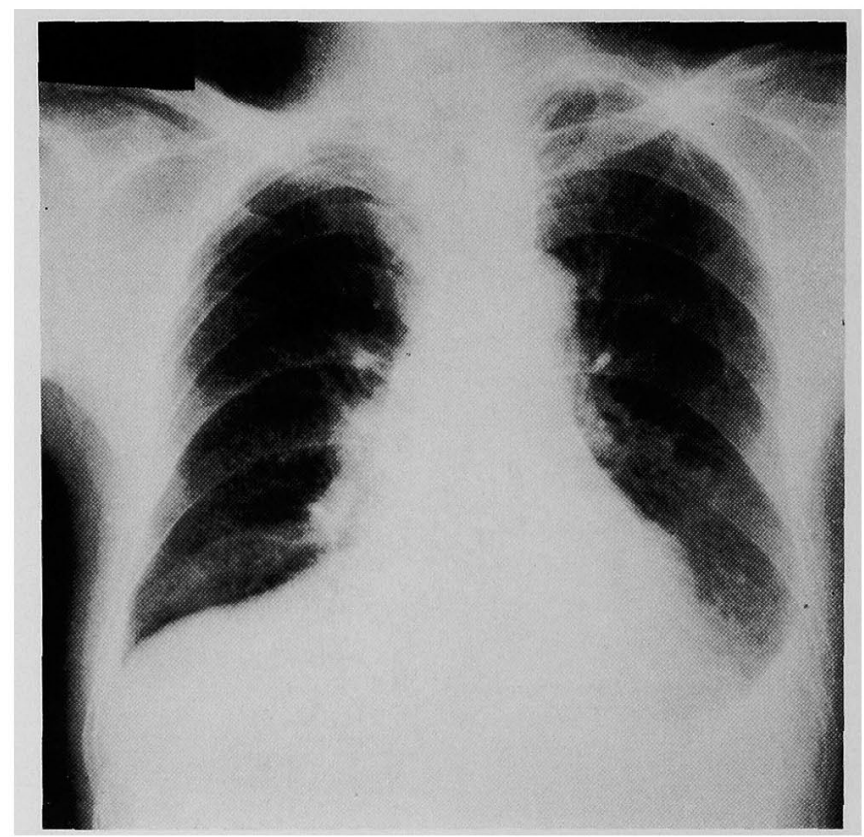

Fig. 10. Chest roentgenogram in recovery phase (Nov., 1976).

ible with the echocardiographic findings.

The theory proposed by Dornhorst et $\mathrm{al}^{3)}$ and subsequently supported by Shabetai et $\mathrm{al}^{4)}$ and Ruskin et $\mathrm{al}^{5 \text { ) }}$ is the right heart changes by respiration. According to these authors, despite the presence of cardiac tamponade, the normal inspiratory increase in right ventricular filling is preserved, and this in turn results in slight elevation of the intrapericardial pressure, causing some degree of left ventricular compression. D'Cruz et al ${ }^{8)}$ also demonstrated cyclic changes of the ventricular dimensions and DDR of the mitral valve, and the diminished change of ventricular dimension seems to be reasonable to occur in this occasion. The reason why the present study could not disclose the marked cyclic changes in the left and right ventricular end-diastolic dimensions is explained in the following manner. During inspiration, the ejection time is short mainly due to delayed opening of the aortic valve without definite change in Q-IIA interval, thus the end-systolic ventricular volume is relatively large, and the subsequent diastolic filling is small and does not necessarily require the mitral inflow during the rapid filling phase. Only the atrial systole is sufficient to fill the ventricle. During expiration, the ejection time is longer and the end-systolic volume is relatively small, thus the filling during all phases of diastole ensues unless the diastolic expansion of the ventricle is suppressed by the effusion. If the heart swings (pendular motion), the direction of echo beam should be changed from beat to beat, 


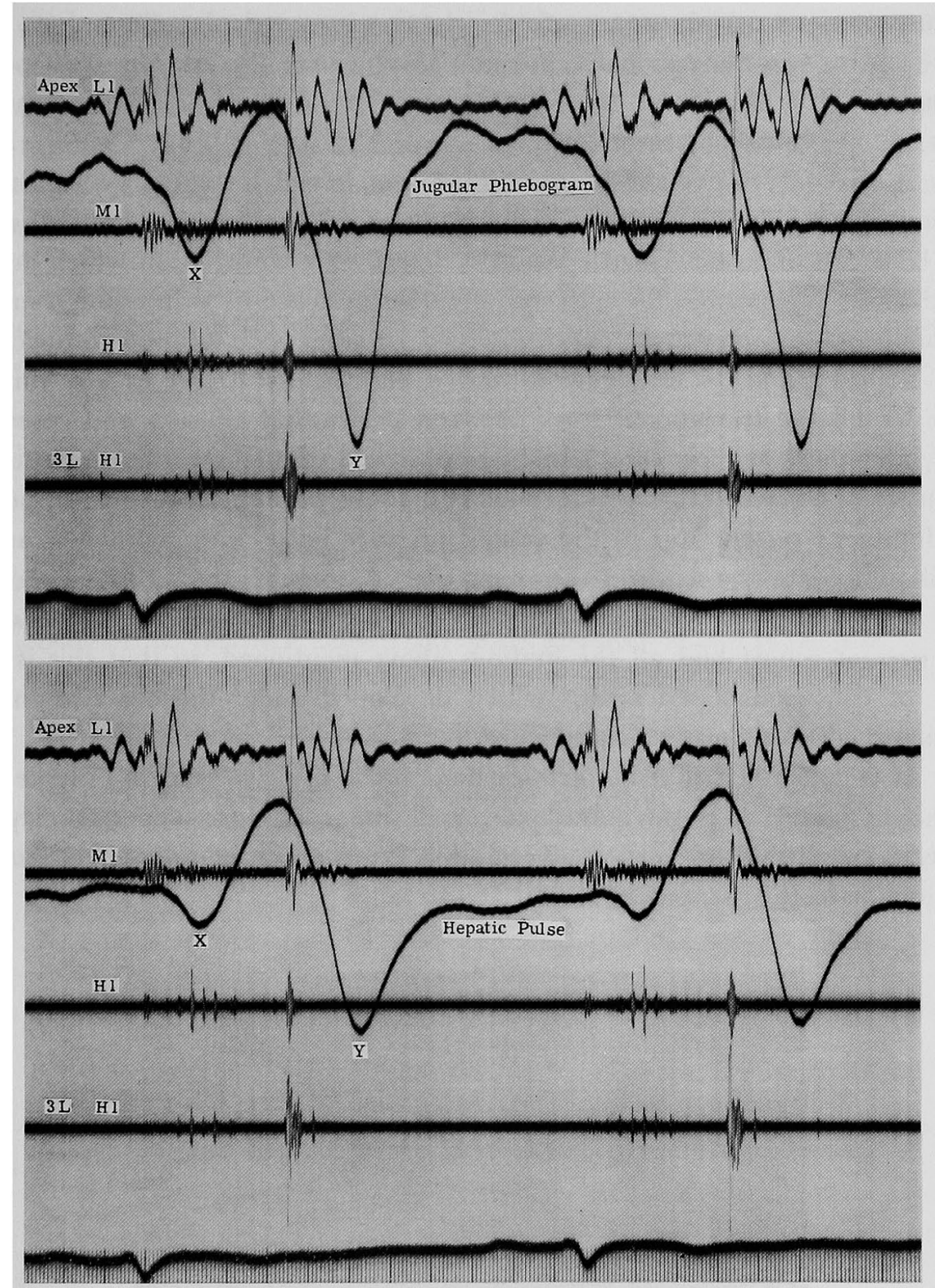

Fig. 11. Phonocardiogram, jugular phlebogram and hepatic pulse tracing in recovery phase (Nov., 1976). All graphic data indicate the typical evidence of constrictive pericarditis.

giving rise to the marked change in the ventricular dimension merely due to the positional change of the heart. Without manifest pendular motion, however, such a technical problem needs not be considered as in our case. Unfortunately, however, the presence of the paradoxical motion of interventricular 
septum does not permit to measure the changes in ventricular dimensions. In this respect, two-dimensional echocardiography may disclose the real mechanism of pulsus paradoxus by observing more accurate change in the ventricular volume caused by respiration.

A mixed "effusive-constrictive" condition, in which there is a constriction of the heart by the visceral pericardium in the presence of the tense effusion, has been less often described. Wood ${ }^{13}$ described a constriction which developed under observation for active tuberculous pericarditis with effusion in the subacute stage. Hancock ${ }^{14)}$ also observed 13 cases with effusion-constriction and named subacute effusive-constrictive pericarditis. It is usually impossible to tell the transitional stage between pericardial effusion and constriction, and there are few cases which are observed throughout the whole stage. Hancock ${ }^{14)}$ described that enlarged heart shadow, prominent pardoxical pulse, predominant systolic dip in the venous pressure pulse, and absence of atrial fibrillation were the features that tended to distinguish the effusive-constrictive cases from those with non-effusive constrictive pericarditis. The case in the present report had all the findings above-mentioned, so that this is the case of constrictive pericarditis which developed under the observation for subacute effusive-constrictive pericarditis.

It is concluded that the echocardiogram may be helpful to observe the pathophysiology of pulsus paradoxus, and that respiration-dependent cyclic changes in mitral and aortic valve motion are pathognomonic sign of pulsus paradoxus in this particular case.

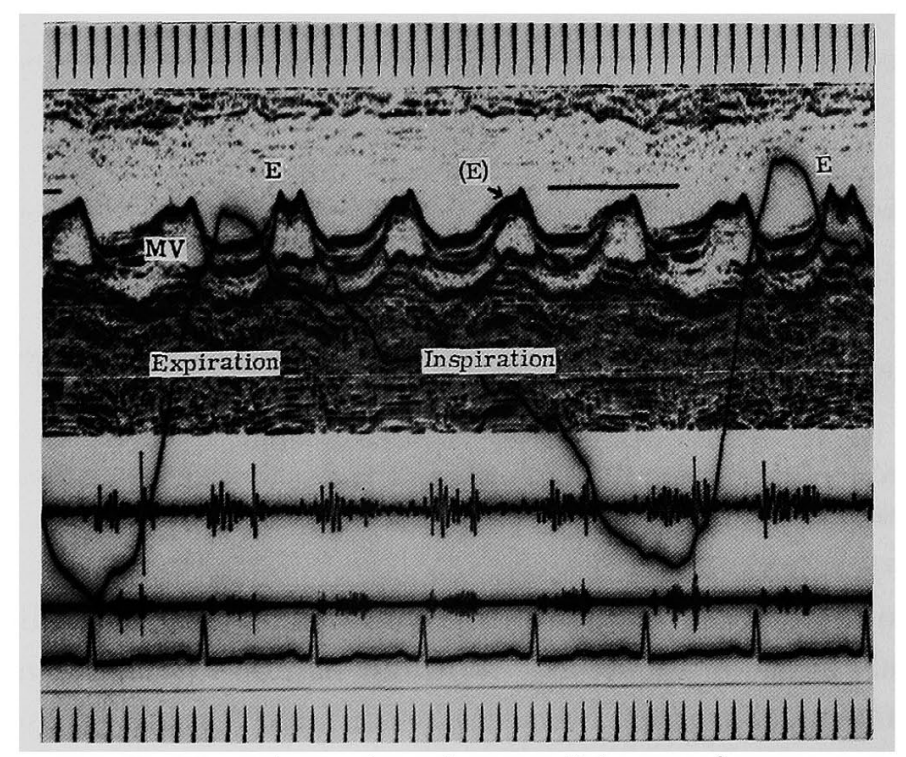

Fig. 12. Mitral valve echogram of the second case. 
Addendum: Since this paper was submitted for publication, we had a chance to study the second case (68-year-old female), in which the insufficient mitral valve opening manifested by the round or flat and incomplete $\mathrm{E}$ wave during inspiration (Fig. 12). This incomplete opening during rapid filling phase is forme fruste in comparison with the first case in this report. The behavior of the aortic valve was almost identical.

These 2 cases were altogether presented at the 22nd Annual Meeting of the American Institute of Ultrasound in Medicine (AIUM), Dallas, Texas, November 1977, and appeared in abstract form in the Proceeding p. 108 (No. 137).

\section{REFERENCES}

I. Katz LN, Gauchat HW: Observations on pulsus paradoxus (with special reference to pericardial effusion). II. Experimental. Arch Intern Med 33: 371, 1924

2. Golinko RJ, Kaplan N, Rudolph AM: The mechanism of pulsus paradoxus during acute pericardial tamponade. J Clin Invest 42: 249, 1963

3. Dornhorst AC, Howard P, Leathart GL: Pulsus paradoxus. Lancet 1: 746, 1952

4. Shabetai R, Fowler NO, Fenton JC, Masangkay M: Pulsus paradoxus. J Clin Invest 44: 1882, 1965

5. Ruskin JC, Bache RJ, Rembert JC, Greenfield JG: Pressure flow studies in man. Effect of respiration on left ventricular stroke volume. Circulation 48: 78, 1973

6. Wood P: Diseases of the Heart and Circulation. JB Lippincott, London, 1952

7. Dock W: Inspiratory traction on the pericardium. Arch Intern Med 108: 837, 1961

8. D'Cruz IA, Gohen HC, Prabhu R, Glick G: Diagnosis of cardiac tamponade by echocardiography. Changes in mitral valve motion and ventricular dimensions, with special reference to paradoxical pulse. Girculation 52: 460, 1975

9. Feigenbaum H, Waldhausen JA, Hyde LP: Ultrasound diagnosis of pericardial effusion. JAMA 191: 107, 1965

10. Feigenbaum H: Echocardiographic diagnosis of pericardial effusion. Am J Cardiol 26: 475, 1970

11. Horowitz MS, Schulta CS, Stinson EB, Harrison DC, Popp RL: Sensitivity and specificity of echocardiographic diagnosis of pericardial effusion. Circulation $\mathbf{5 0}: 239,1974$

12. Gibson TC, Grossman W, McLaurin LP, Moos S, Craige E: An echocardiographic study of the interventricular system in constrictive pericarditis. Brit Heart J 38: 738, 1976

13. Wood P: Chronic constrictive pericarditis. Am J Cardiol 7: 48. 1961

14. Hancock EW: Subacute effusive-constrictive pericarditis. Circulation 43: 183, 1971 